\title{
FERIAS TEMÁTICAS: JUEGO, ESPACIOS Y MATERIALES COMO RECURSOS DE MOTIVACIÓN Y GLOBALIZACIÓN DE LOS CONTENIDOS CURRICULARES EN LA ETAPA DE INFANTIL
}

\author{
THEMED EVENTS: GAME, SPACE AND MATERIALS AS \\ RESOURCES MOTIVATION AND GLOBALIZATION \\ CURRICULUM CONTENT ON THE STAGE OF CHILDREN
}

Recibido: 10/04/2015

Revisado: 30/04/2015

Aceptado: 26/05/2015

Rosario Padial Ruz

La etapa de educación infantil es la etapa más importante en el desarrollo global e integral del niño/a.

Teniendo en cuenta que la primera forma de conocimiento que se produce en este es a través de su cuerpo, la formación e innovación en metodologías de intervención didáctica en el ámbito motor van a ser fundamentales para un buen desarrollo cognitivo, físico, social y emocional en el alumnado.

Esto supone una necesidad de dotar de buenos especialistas a esta etapa educativa, basada no solo en la formación docente, sino también en la investigación en el aula.

En el campo de la educación física y su papel en esta etapa desde una perspectiva global e interdisciplinar, es necesaria una buena formación del docente en metodologías que permitan el desarrollo integral del alumnado, basadas en un enfoque constructivista y prestando especial atención en el desarrollo práctico de estrategias de enseñanza basadas en el cuerpo y el movimiento.

Esto nos lleva a la necesidad de seguir una metodología de formación en didáctica, desde un modelo de investigación-acción, basado en la utilización de recursos como los contenidos motrices, los juegos, la expresión corporal, los espacios y los materiales, para la innovación en estrategias de intervención en el ámbito escolar que permitan un proceso de enseñanza-aprendizaje global e interdisciplinar de todos los contenidos curriculares de la etapa.

En esta línea, "la feria temática" es una de las estrategias de intervención que, desde las asignaturas que imparto en la E.U. de Magisterio y la Facultad de Ciencias de la Educación de Granada, vengo desarrollando en colaboración con diferentes Escuelas Infantiles de Granada, desde la asignatura "Técnicas de educación motriz en edades tempranas", asignatura de $2^{\circ}$ curso educación infantil y actualmente, optativa del Grado de Maestro en Educación Infantil.

Esta actividad pretende, por un lado contribuir al desarrollo de los contenidos más cognitivos y actitudinales del currículo, así como desarrollar la motricidad de los niños/as y crear hábitos de actividad física en estas edades que contribuyan a la mejora de su salud.

\section{Importancia de la motricidad en el desarrollo del niño}

Todos los aprendizajes que se producen en el ser humano desde el nacimiento, e incluso antes del mismo (en el tercer trimestre de gestación del feto), se realizan a través del cuerpo y del movimiento.

El ser humano, por medio del movimiento, aprende a conocerse a sí mismo, al medio que le rodea, comunicarse con él, a relacionarse con los demás. En definitiva, se desarrolla en todos sus ámbitos: cognitivo, afectivo, social y psicomotor (Cuevas y Gil, 2013; Gil, Contreras, Gómez, 2008; Moreno, López, Gutiérrez, Cascada y Fernández, 2004).
Son numerosos los autores que han escrito y basado sus trabajos en la importancia de la motricidad en la construcción y desarrollo de la personalidad del niño/a en edades tempranas (Piaget 1968, 1969; Wallon, 1980; Gesell, 1958; Freud, 1968; Bruner, 1979; Guilmain, 1981; Ajuriaguerra, 1978; Le Boulch, 1981; Vayer, 1973; Da Fonseca, 1984, 1988 y 1996; Cratty, 1990 y Gallahue, 1985).

En el campo de la pedagogía, Moreno, López, Gutiérrez, Cascada y Fernández (2004), destacan a Rousseau, Pestalozzi y Montessori, como los autores más representativos que consideraron vital la Educación de lo corporal en el proceso de formación de la personalidad del niño/a.

\section{La motricidad en el currículo de la etapa}

La importancia del juego y del movimiento viene recogida a través del currículo de la etapa (Orden $\mathrm{ECl} / 3960 / 2007$, de 19 de diciembre, por la que se establece el currículo y se regula la ordenación de la educación infantil):

Artículo 2. Principios generales.

3. La intencionalidad educativa debe orientar en esta etapa todos los momentos, actividades y situaciones escolares. Las distintas propuestas y experiencias de aprendizaje se abordarán desde un enfoque integrado y globalizador.

4. Los métodos de trabajo en ambos ciclos se basarán en las experiencias, en la actividad infantil y en el juego, y se aplicarán en un ambiente de seguridad, afecto y confianza para potenciar la autoestima y la integración social.

Artículo 3. Fines de la educación infantil:

Hace referencia al desarrollo integral del niño/a como finalidad principal de la etapa.

"La finalidad de la Educación infantil es la de contribuir al desarrollo físico, afectivo, social e intelectual de los niños y las niñas".

Artículo 4. Objetivos.

1. La Educación infantil contribuirá a desarrollar enlos niños y las niñas las capacidades que les permitan:

a) Conocer su propio cuerpo y el de los otros, sus posibilidades de acción y aprender a respetar las diferencias.

c) Adquirir progresivamente autonomía en sus actividades habituales.

d) Desarrollar sus capacidades afectivas.

e) Relacionarse con los demás y adquirir progresivamente pautas elementales de convivencia y relación social, así como ejercitarse en la resolución pacífica de conflictos.

f) Desarrollar habilidades comunicativas en diferentes lenguajes y formas de expresión.

g) Iniciarse en las habilidades lógico-matemáticas,en la lectoescritura y en el movimiento, el gesto y el ritmo. 
Artículo 5. En cuanto al tratamiento de las áreas indica que:

2. Estas áreas deben entenderse como ámbitos propios de la experiencia y el desarrollo infantil y del aprendizaje de actitudes, procedimientos y conceptos, que contribuirán al desarrollo de los niños y las niñas y propiciarán una primera aproximación a la interpretación de su entorno y a la atribución de significados, facilitando su participación activa en él.

3. Los contenidos de la Educación infantil se abordarán por medio de propuestas integradas que tengan interés y sean significativas.

4. En el primer ciclo se atenderá especialmente a la adquisición de hábitos elementales de salud y bienestar, a la mejora de sus destrezas motrices y de sus habilidades manipulativas, al desarrollo del lenguaje, al establecimiento de vínculos afectivos con los demás y a la regulación progresiva de la expresión de sentimientos y emociones.

Atendiendo a esto, la metodología que se sugiere en el anexo II de dicho documento, se basa en los principios pedagógicos que se establecen para esta etapa: enfoque globalizador de los contenidos, enseñanza significativa, utilización del juego como instrumento principal, principio de actividad (observación y experimentación), utilización de espacios y materiales para la consecución de las intenciones educativas. "Los métodos de trabajo en ambos ciclos se basarán en las experiencias, las actividades y el juego".

Como se puede observar a través del currículo de la Etapa, el desarrollo físico y motor es uno de los ejes centrales mediante el cual giran los diferentes aprendizajes que deben desarrollar el niño/a. La etapa de educación infantil, es una etapa de continuo movimiento que debe ser canalizado por el educador mediante una metodología basada en el juego, éste se presenta ante el niño/a otorgando infinidades de experiencias y vivencias que le llevarán a adquirir aprendizajes de un modo muy motivador y a la vez significativo para é (Moreno, López, Gutiérrez, Cascada y Fernández, 2004). El juego y la actividad física serán instrumentos de trabajo a partir del cual se irán incardinando todos los contenidos del currículo.

Por tanto, trabajar las distintas áreas a través del cuerpo y el movimiento responde a la necesidad de una enseñanza global, interdisciplinar y significativa para los niños/as (Conde y Viciana, 1997; Gil, 2003; Llorca y Vega, 1998; Mendiara, 1997; Orden ECl/3960/2007, de 19 de diciembre; Real Decreto 1630/2006, de 29 de diciembre; Ruiz, 2003 y Vaca, 1995).

Ferias Temáticas: juego, espacios y materiales como recursos de motivación y globalización de los contenidos curriculares en la Etapa de Infantil

En la propuesta que expongo a continuación y que he denominado "FERIA TEMÁTICA", se pretenden aunar diversos recursos utilizados en el campo motor con el objeto de poder desarrollar de forma global e interdisciplinar los diferentes ámbitos del desarrollo humano y los contenidos que forman las tres áreas de esta Etapa.

Los recursos utilizados, siguiendo a Gil, Contreras y Gómez, (2008) son:

- El juego y la interacción motriz entre los compañeros y los adultos como el principal recurso didáctico.

- La organización del espacio y de los materiales como la principal estrategia de intervención didáctica.

Ambos recursos responden a la necesidad en esta etapa de trabajar, como indica el currículo, desde la acción y la experimentación.

Es una propuesta que, siguiendo a Gil, Contreras y Gómez (2008); Moreno, López, Gutiérrez, Cascada y Fernández (2004) y Pastor (2013), respeta principios básicos propios de la etapa como son:

- Principio de enseñanza global.

- Propuesta de actividades variadas y atractivas que cumplan siempre el principio de globalidad.

- Utilización de refuerzos encaminados a garantizar una adecuada motivación hacia la práctica
- Utilización de recursos espaciales y materiales que permitan la motivación y consecución de los objetivos propuestos.

- Utilización de una metodología lúdica, basada en el juego y el movimiento, para poder globalizar e interdisciplinar los contenidos curriculares de la etapa.

Según cómo se organice la circunstancia ambiental se puede potenciar la aparición de comportamientos específicos. En consecuencia, la manipulación intencionada de ambientes de aprendizaje mediante la organización de espacios y materiales puede implicar a los niños/as en conductas motrices concretas que respondan a los objetivos planteados. De la misma forma que la acción, la experimentación, el juego y la interacción de los niños/as con sus compañeros y con el adulto, en un ambiente distendido y afectuoso, son factores y recursos que cumplen un papel esencial para que pueda producirse el crecimiento personal (Gil, Contreras y Gómez ,2008).

Basándonos en estas premisas anteriores, la feria temática tiene como objetivo la utilización de espacios y materiales con el fin de desarrollar no solo la motricidad, sino contribuir al desarrollo integral del alumnado, desarrollando además los ámbitos cognitivo, afectivo y social-relacional, además de motivar hacia la actividad física como base de mejora de la salud.

El concepto de Feria Temática está basado en el concepto de Feria del Juego. Según Ruiz y cols (1998), podemos definir la feria del Juego como "el conjunto de actividades lúdicas o juegos en la que cada uno de ellos se organiza en stands independientes".

Collado y Cadena (2015), la definen como el "cómputo de actividades, tareas o juegos desde una vertiente lúdica en la que cada uno de éstos está ubicado y estructurado en el espacio por medio de un stand. Cada juego deberá tener una vertiente lúdica y educativa".

Los participantes podrán ir de juego a otro, según sus preferencias, sin tener que guardar un orden preestablecido, pero teniendo que participar al final de la sesión en todas las actividades (Collado y Cadena ,2015y Ruiz y cols, 1998). En el caso de la feria temática y debido a que los niños/as son muy pequeños, los cambios de actividad se realizarán de forma controlada y organizada.

En la feria temática, el eje central, entorno al se establecen los juegos, los espacios y materiales, podrá ser cualquier contenido del currículo de la etapa. Este contenido se trabajará de forma global e interdisciplinar desde el ámbito motor, utilizando el cuerpo y el movimiento como herramientas fundamentales. Es muy importante, para captar la atención y la motivación del alumnado hacia la actividad, la preparación del espacio y del material, que deberá estar relacionado con el eje central de trabajo de la feria y ser llamativo y atrayente para el alumnado, como se verá en la propuesta que se desarrolla a continuación.

En el ejemplo que se expone a continuación, se realizó una feria temática para animación a la lectura y el conocimiento de los cuentos populares tradicionales infantiles (Padial y Sáez-López, 2014).

En todas las actividades se trabaja el contenido central y el desarrollo de los contenidos psicomotores propios del $2^{\circ}$ ciclo de Educación Infantil, cumpliendo con los principios de globalización, interdisciplinariedad y utilización del espacio, los materiales y la actividad motriz como recursos para la motivación y aprendizaje significativo del alumnado.

\section{REFERENCIAS BIBLIOGRÁFICAS}

Ajuriaguerra, J. (1978). Manual de psiquiatría infantil. Barcelona, Científico-Médica.

Bruner, J. (1979). Algunos elementos del descubrimiento. En L. Shulman \& E. Keislar (Eds.), Aprendizaje por Descubrimiento. Evaluación Crítica (pp. 115-132). México D.F.: Trillas. 
Collado, D. y Cadenas, C. (2015). La feria del juego como recurso didáctico. Granada. Fleming.

Conde, J. L. y Viciana, V. (1997). Fundamentos para el desarrollo de la educación física en edades tempranas. Málaga: Aljibe

Cratty, B. J. (1990). Desarrollo perceptual y motor en los niños. Barcelona: Paidós.

Cuevas, R. y Gil, P. (2013). La planificación educativa de la Educación Física en Educación Infantil: la perspectiva globalizadora. En Gil, Pedro. Desarrollo curricular de la Educación Física en la Educación Infantil (pp57-68). Madrid: Pirámide.

Fonseca Da, V. (1984). Filogénesis de la motricidad. Madrid, García Núñez.

Fonseca Da, V. (1988). Ontogénesis de la motricidad. Madrid, García Núñez.

Fonseca Da, V. (1996). Estudio y génesis de la psicomotricidad. Barcelona: Inde.

Freud, S. (1968). Obras completas. Vol. III. Madrid: Biblioteca Nueva.

Gallahue, D.; MCclenaghaN, B. (1985). Movimientos fundamentales. Buenos Aires: Panamericana.

Gesell, A. (1958). Psicología evolutiva de 1 a 16 años. Vol. II. Buenos Aires: Paidós.

Gil,P. (2003): Diseño y desarrollo curricular en Educación Física y Educación Infantil. Sevilla: Wanceulen.

Gil, P., Contreras, O., Díaz, A. y Lera, A. (2006). La educación física en su contribución al proceso formativo de la educación infantil. Revista educación, 339, 401-433. www.revistaeducacion.mec.es/re339/re339_18.pdf

Gil, P. Contreras, O. y Gómez- Barreto, I. (2008). Habilidades motrices en la infancia y su desarrollo desde una educación física animada. Revista iberoamericana de educación, 47, 71-96. http://www.rieoei.org/rie47a04.pdf

Guilmain, E. (1981). Evolución psicomotriz desde el nacimiento a los 12 años. Barcelona, Médica y técnica.

LeBoulch, J. (1981). La educación por el movimiento en la Educación Escolar. Barcelona: Paidós.

LLorca, M.; Vega, A. (1998): Psicomotricidad y globalización del currículo de la Educación Infantil. Málaga: Aljibe.

Mendiara, J. (1997): Educación Física y aprendizajes tempranos. Contribución al desarrollo global de los niños de 3 a 6 años y estudio de sus estrategias de aprendizaje en espacios de acción y aventura. Tesis doctoral inédita. Zaragoza, Universidad de Zaragoza.

Moreno, J.A., López, B., Gutiérrez, EM, Cascada, M. y Fernández, MR. (2004). Situación actual de la educación física en la etapa de 0 a 6 años según el profesorado de educación infantil. Revista Iberoamericana de Psicoeducación física y técnicas corporales, 4(16), 17-34. http://www.iberopsicomot.net/2004/num16/16articulo2.pdf

Orden ECl/3960/2007, de 19 de diciembre, por la que se establece el currículo y se regula la ordenación de la educación infantil.

Padial, R. y Sáez-López, P. (2014). Los cuentos populares/tradicionales en educación infantil. Una propuesta a través del juego. Revista de Educación, Motricidad e Investigación,[S.I.],n.2,p.32-47. Disponible en:

<http://www.uhu.es/publicaciones/ojs/index.php/e-motion/article/view/2375>. Fecha de acceso: 07 may. 2015

Pastor, J.C. (2013). La clase y la organización de las actividades de enseñanza-aprendizaje de Educación Física en Educación Infantil. En Gil, Pedro. Desarrollo curricular de la Educación Física en la Educación Infantil (pp44-53). Madrid: Pirámide.

Piaget, J. (1968): Seis estadios de psicología. Madrid: SeixBarral.

Piaget, J. (1969): Biología y crecimiento. Madrid, Siglo XXI.

Real Decreto 1630/2006, de 29 de diciembre, por el que se establecen las enseñanzas mínimas del segundo ciclo de Educación infantil.

Ruiz , F. (2003): Proyecto docente: desarrollo psicomotor. Trabajo inédito. Almería, Universidad de Almería.

Ruiz, L., y cols (1998). "La Feria del juego. Una propuesta globalizadora". Publicaciones de la facultad de educación y humanidades del campus de Melilla. Melilla, Universidad de Granada, 28.

Vaca, M. (1995): Tratamiento pedagógico de lo corporal en Educación Infantil. Propuesta de un modelo de intervención a través del estudio de un caso en el Segundo Ciclo. Tesis doctoral inédita. Madrid, UNED.

Vayer, P. (1973): El niño frente al mundo. Barcelona: Científico Médica.

Wallon, H. (1980): La evolución psicológica del niño. Barcelona: Crítica.

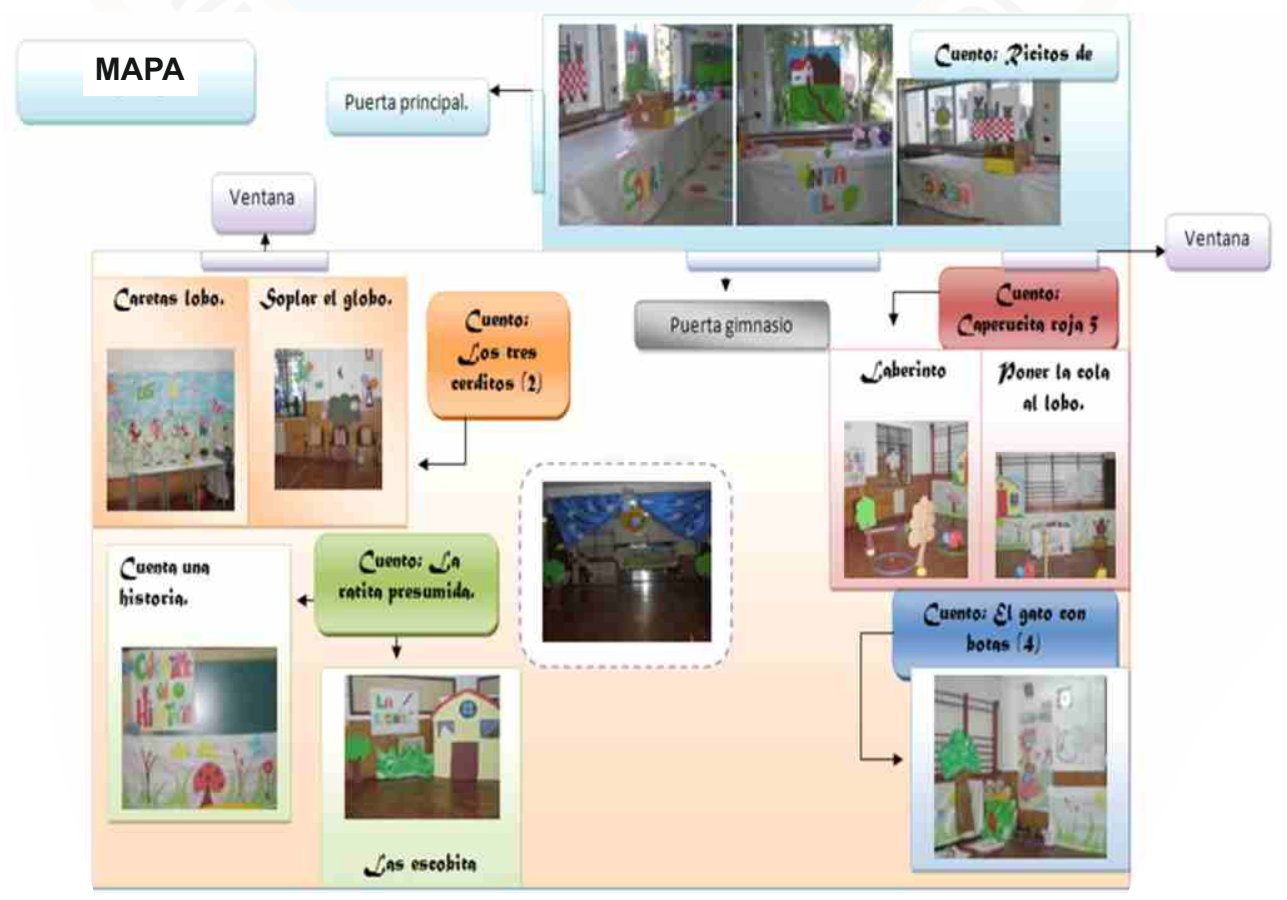

Figura 1. Distribución del espacio y las actividades. 